\title{
Estudio cuantitativo del consumo de drogas y factores sociodemográficos asociados en estudiantes de una universidad tradicional chilena
}

'Departamento de Farmacología, Facultad de Ciencias Biológicas. ${ }^{2}$ Departamento de Fisiología, Facultad de Ciencias Biológicas. ${ }^{3}$ Departamento de Sociología, Facultad de Ciencias Sociales, Universidad de Concepción. Concepción, Chile. ${ }^{a} Q u i ́ m i c a-F a r m a c e ́ u t i c a, P h D$. bProfesor de Biología y Ciencias Naturales, Magíster en Ciencias, PhD. 'Sociólogo, Magister@ en Investigación Social y Desarrollo.

Recibido el 17 de agosto de 2010, aceptado el 6 de mayo de 2011.

Correspondencia a: M. Jacqueline Sepúlveda C. Edmundo Larenas 234, Concepción, Chile. Fono: 56-41-2203894, $56-41-2204243$

Fax: 56-41-2255975 E-mail: jsepulve@udec.cl

\author{
M. JACQUELINE SEPÚLVEDA C. ${ }^{1, a}$, JORGE ROA S. ${ }^{2, b}$, MARCOS MUÑOZ R.,
}

\section{Prevalence of the drug consumption among Chilean university students}

Background: Drug abuse is one of the main public health problems and mostly involves young people. Aim: To determine the prevalence of drug consumption among university students and its associated social factors. Material and Methods: A questionnaire exploring consumption of drugs and social-demographic, economic and religious variables, was applied to 1577 third year students aged $22 \pm 2$ years (54\% females) of a public university. Results: Sixty eight percent of the students were of middle socioeconomic class, $71 \%$ belonged to some religion and $29 \%$ declared themselves as agnostic. Ninety six percent drank alcohol at least once and $29 \%$ never smoked. Among illicit drugs, marijuana was consumed by $22 \%$ followed by cocaine in $5.3 \%$, hallucinogenic drugs in $2.6 \%$, inhalants in $2.3 \%$ and cocaine free base in $1.4 \%$. Six percent consumed stimulants and $13.8 \%$, sedatives. A higher economic income facilitated drug consumption and the adscription to a religion was protective against it. Conclusions: Alcohol is the most commonly consumed drug, and is the substance that generates more problems to students.

(Rev Med Chile 2011; 139: 856-863).

Key words: Alcohol-related disorders; Education, students; Substance-related disorders.
E 1 abuso de drogas es uno de los principales problemas de salud pública en el mundo.

En Chile, desde el año 1994, se realizan estudios por el Consejo Nacional para el Control de Estupefacientes (CONACE), que demuestran la magnitud del problema. El mayor consumo se concentra entre los 19 y 25 años. En este grupo etario, el consumo de alcohol y tabaco, aumenta, alcanzando en el año 2008 prevalencias de vida de $87,2 \%$ y $77,2 \%$ respectivamente. Con respecto a marihuana, pasta base y cocaína, las prevalencias de vida del año 1994 aumentaron de 21,3\% a $42,2 \%$, de $3,2 \%$ a $4,1 \%$ y de $4,8 \%$ a $9,4 \%$ respectivamente, en el año $2008^{1}$.

El inicio del consumo de drogas se produce en los primeros años de la adolescencia y se relaciona con aspectos familiares, socioeconómicos, cognitivos y emocionales.

Un estudio con adolescentes demostró una prevalencia de consumo de cigarrillos de 5,4\%, de alcohol de $37,3 \%$ y de $37,8 \%$ para drogas ilícitas, siendo la influencia de sus pares la principal motivación del consumo ${ }^{2}$.

Una investigación realizada con estudiantes de la Universidad de São Paulo, demostró 86\% de prevalencia de vida de alcohol y una edad promedio de inicio de consumo de 13 años, seguido por tabaco con $65 \%$ y marihuana con $28,5 \%$. Además, se demostró que las mujeres beben dentro de los límites de bajo riesgo, mientras los hombres tienen consumos mayores y presentan conductas riesgosas asociadas al consumo ${ }^{3}$. 
Otro estudio realizado en la University College de Dublín, evaluó la evolución del consumo de drogas, durante 29 años (1973-2002), demostrando una disminución de la prevalencia de consumo de tabaco de $28,8 \%$ a $9,2 \%$ y un fuerte incremento de prevalencia de consumo de alcohol de 74,6\% a $82,5 \%{ }^{4}$.

Una investigación de la Universidad de Zaragoza, demostró que el 17,3\% fuma tabaco diariamente y $13,5 \%$ ocasionalmente. Además reportan que la prevalencia de tabaquismo es mayor en mujeres $(33 \%)$ que en los varones $(27 \%)$. Con respecto al consumo de alcohol, el 66,2\% ha abusado de esta droga, afirmando que se han emborrachado alguna vez en la vida. Al respecto, los investigadores demostraron una directa relación entre el abuso de alcohol y consumo de tabaco, indicando que los fumadores dicen haberse emborrachado al menos una vez en su vida en mayor proporción que los no fumadores. Los resultados de drogas ilícitas, demuestran que la marihuana es la sustancia más consumida por los estudiantes, indicando que $16,5 \%$ la fuma a diario, ocasionalmente o los fines de semana ${ }^{5}$.

A nivel nacional, un estudio en escolares, provenientes de colegios municipalizados o subvencionados, de niveles socioeconómicos medio, medio-bajo y bajo, demostró que la edad de inicio de consumo de alcohol y tabaco es alrededor de los 11 años y las prevalencias de último año y último mes de drogas lícitas fueron de $29 \%$ y $15 \%$ respectivamente. La prevalencia de consumo reciente de drogas ilícitas fue de 3,5\%, y se asoció con el consumo actual y reciente de tabaco y de alcohol ${ }^{6}$.

Otro estudio, en una universidad privada, con estudiantes de nivel socioeconómico medio alto y alto, demostró que el consumo de tabaco y alcohol alcanzan $78,9 \%$ y $80,3 \%$ respectivamente. En el caso de las drogas ilícitas el consumo de marihuana fue de $63,4 \%$, de cocaína $4,9 \%$ y de éxtasis $2,1 \%{ }^{7}$.

Investigaciones en estudiantes universitarios chilenos demostraron que más del $50 \%$, perciben que sus pares consumen tabaco diariamente, alcohol y marihuana semanalmente y cocaína anualmente . $^{8}$

Un estudio entre estudiantes de medicina, demostró que fuman menos y consumen más alcohol que los jóvenes chilenos y fuman más y consumen menos alcohol que estudiantes de medicina de otros países?.

Con todos estos antecedentes, el objetivo de este estudio fue identificar las prevalencias, los factores sociales y los riesgos asociados al consumo de drogas en estudiantes de una universidad tradicional chilena.

\section{Material y Método}

Se realizó un estudio exploratorio transversal cuantitativo en el año 2007, con 1.577 estudiantes. La encuesta se hizo en los tres Campus de la Universidad, incluyendo 15 Facultades y 59 carreras. Se aplicó un cuestionario diseñado y validado por CONACE, agregando preguntas académicas (carrera, año cursado). Esta nueva versión del cuestionario fue piloteado con una sub-muestra cercana al $10 \%$ de la muestra total para validar las incorporaciones. La variable dependiente fue Prevalencia de Consumo de drogas y las variables independientes fueron edad, situación socioeconómica, religión y carrera.

La muestra fue de tipo no probabilística, un tipo de muestra caracterizada por la presencia del juicio personal del muestrista en la estrategia de selección de elementos ${ }^{10}$.

La encuesta fue aplicada por un grupo de estudiantes, previamente entrenados en el proceso de recolección de información a través de encuestas.

Tanto los profesores de los cursos encuestados como las autoridades de todas las facultades de la universidad fueron anteriormente informados de la aplicación de los instrumentos confirmando fecha y hora de su aplicación. La encuesta fue aplicada con consentimiento informado oral, garantizando voluntariedad y confidencialidad en la aplicación.

\section{Resultados}

\section{Caracterización social}

De la población encuestada el 54,3\% correspondió a mujeres y el $45,6 \%$ a hombres. La edad

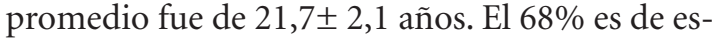
tratos medios (Q2, Q3 y Q4) (Figura 1). El 71,3\% pertenece a alguna religión y $28,7 \%$ se declara agnóstico, libre pensador o no pertenecer a alguna religión (Figura 2).

\section{Prevalencias y edad de inicio de consumo}

La Tabla 1 indica las prevalencias de vida, de último año y la edad promedio de inicio de consumo de los encuestados. Las drogas lícitas no sólo 
Consumo de drogas en universitarios - M. J. Sepúlveda et al
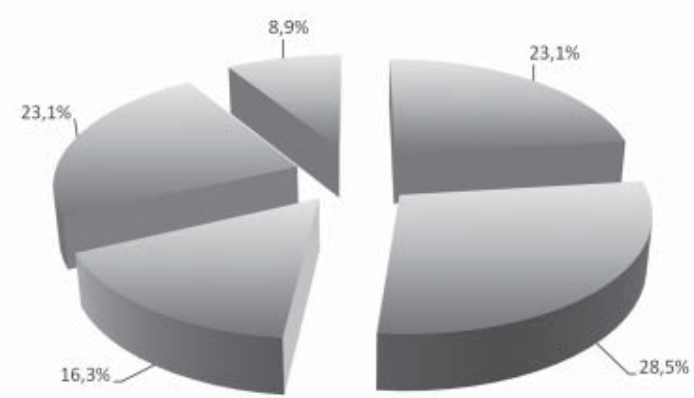

Figura 1. Distribución de ingresos socioeconómicos familiares de los encuestados. Los ingresos socioeconómicos se clasificaron de acuerdo a: Quintil 1 (Q1): Estudiantes pertenecientes a familias que perciben ingresos entre $\$ 120$ mil y $\$ 245$ mil. Quintil 2 (Q2): Estudiantes pertenecientes a familias que perciben ingresos entre $\$ 245$ mil a $\$ 440$ mil. Quintil 3 (Q3): Estudiantes pertenecientes a familias que perciben ingresos entre $\$ 440$ mil a $\$ 670$ mil. Quintil 4 (Q4): Estudiantes pertenecientes a familias que perciben ingresos entre $\$ 670$ mil a $\$ 1,8$ millones. Quintil 5 (Q5): Estudiantes pertenecientes a familias que perciben ingresos de $\$ 1,8$ millones a $\$ 8$ millones.

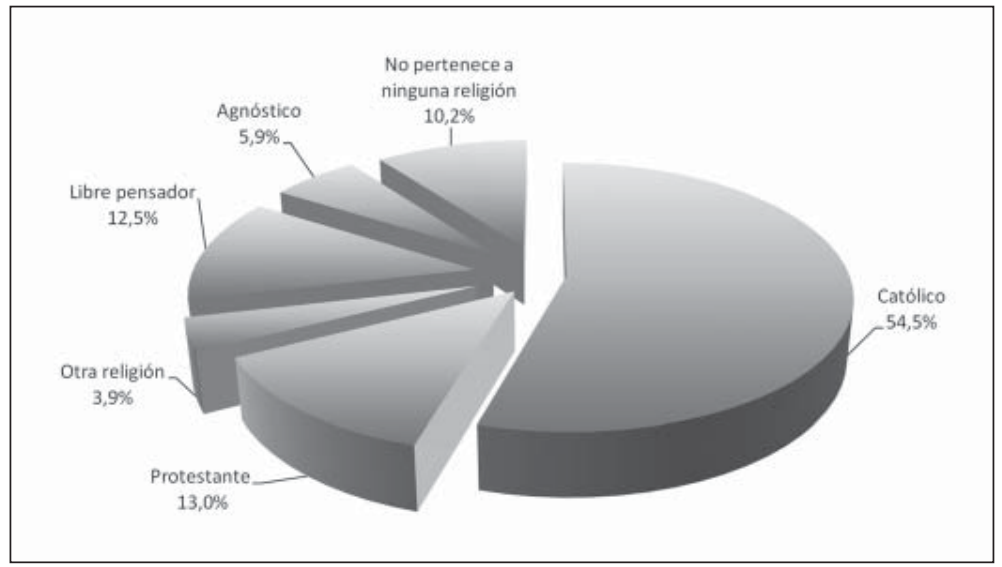

Figura 2. Distribución por religión del encuestado.

Tabla 1. Prevalencia de vida, de último año y edad promedio de inicio de consumo

\begin{tabular}{|lccccccc|}
\hline Substancia & $\begin{array}{c}\text { Edad de } \\
\text { inicio }\end{array}$ & $\begin{array}{c}\text { Prevalencia de vida de población } \\
\text { encuestada (\%) }\end{array}$ & \multicolumn{3}{c|}{$\begin{array}{c}\text { Prevalencia último año de } \\
\text { población encuestada (\%) }\end{array}$} \\
Alcohol & 15 & 95,4 & 97,7 & 96,4 & 80,3 & 87.6 & Total \\
Tabaco & 15 & 69,2 & 72,6 & 71 & 39,1 & 40.1 & 39.6 \\
\hline Marihuana & 18 & 36,0 & 54,8 & 44,1 & 15 & 30 & 22 \\
Cocaína & 19 & 2,0 & 9,3 & 5,3 & 1,0 & 2.6 & 1.7 \\
\hline Pasta Base & 19 & 0,4 & 2,6 & 1,4 & 0,4 & 1.0 & 0.7 \\
\hline Éxtasis & 20 & 0,2 & 1,6 & 0,8 & 0,1 & 0.3 & 0.2 \\
\hline Inhalantes & 15 & 1,8 & 3,0 & 2,3 & 0,1 & 0.4 & 0.3 \\
\hline Alucinógenos & 19 & 1,1 & 4,5 & 2,6 & 0,1 & 0.7 & 0.4 \\
\hline Estimulantes s/r & 18 & 3,4 & 8,5 & 5,7 & 1,4 & 1.7 & 1.6 \\
\hline Tranquilizantes s/r & 18 & 14,5 & 12,9 & 13,8 & 5,9 & 3.3 & 4.7 \\
\hline Analgésicos s/r & 17 & 5,4 & 5,2 & 5,3 & 1.6 & 1.4 & 1.6 \\
\hline
\end{tabular}

$\mathrm{s} / \mathrm{r}=$ Sin receta médica. 
han sido las más probadas por los estudiantes, sino que ellas están presentes desde la adolescencia y no están determinadas por el sexo de los estudiantes.

Casi la totalidad de ellos han probado el alcohol $(96,4 \%)$, no obstante, es necesario destacar que $29 \%$ de los estudiantes no ha probado el tabaco.

Respecto a las drogas ilícitas, la marihuana ha sido la más experimentada, si bien comienzan a probarla en la adolescencia, es en el período de los 18 años a 25 años donde las primeras experiencias con la marihuana llegan al 51\%.

Las experiencias con la cocaína llegan al 5,3\%, sin embargo, entre los 18 y 24 años se presentan el 78,6\% del total de experiencias con la sustancia. Los hombres que han probado la cocaína representan 79,2\% del total de experiencias con la sustancia, por lo que podemos establecer que esta droga está significativamente determinada por el sexo del estudiante.

Los alucinógenos son la tercera droga ilícita más probada por los estudiantes (26\%). Los hombres representan el 76,9\% del consumo total de alucinógenos y las mujeres $23 \%$. Al respecto, el $85,7 \%$ de las mujeres que probaron alucinógenos lo hicieron entre los 18 y 22 años y el 56\% de los hombres señala haberlo probado entre los 18 y 29 años.

Los estudiantes que han probado la pasta base representan el 1,4\% del total de la población estudiantil encuestada. Las mujeres representan el $14,2 \%$ del total de experiencias con la sustancia y los hombres $85,7 \%$, por lo que las experiencias con pasta base se dan preferentemente en círculos sociales masculinos.

El éxtasis es poco probada por los estudiantes $(0,8 \%)$, con $87,5 \%$ de experiencias en hombres y $12,5 \%$ en mujeres. Además, es entre los 20 y 29 años que se desarrolla el mayor número de experiencias con la sustancia $(72,8 \%)$ siendo éstas casi exclusivamente masculinas.

Los estudiantes que señalan haber probado sustancias inhalantes son el $2,3 \%$ respecto a la población total de estudiantes encuestados, con $39,1 \%$ de participación femenina y $60,8 \%$ masculina. Entre los 12 y los 17 años se manifiesta el $73,6 \%$ de las primeras experiencias, período en el cual está representado el 100\% de las experiencias femeninas.

Respecto a las substancias de uso médico, el $5,7 \%$ de los estudiantes señala haber probado estimulantes, con $68,4 \%$ de los hombres y $31,5 \%$ de las mujeres. Entre los 18 y 24 años las primeras experiencias con estimulantes llega a los $63,3 \%$. En este mismo período el $69,3 \%$ de las mujeres que probaron los estimulantes prueba la sustancia, mientras que los hombres alcanzan $42,2 \%$ de experiencias en dicho período.

Los fármacos sin receta médica más probados por los estudiantes son los tranquilizantes $(13,8 \%)$, el 60\% de ellos dicen haberlos consumido entre los 18 y 24 años. En este período de edad las primeras experiencias femeninas con tranquilizantes llegan al $65,3 \%$ y masculinas al 55,8\%. Si agregamos, además, que las mujeres representan el 57,9\% del total de experiencias con la sustancia, se puede decir que a través del uso femenino de los tranquilizantes y de substancias de uso médico se rompe el sostenido dominio masculino sobre las experiencias con drogas en general. La Tabla 2 indica las prevalencias de último año según carrera. Se observa que el consumo de drogas lícitas, alcohol y tabaco, además de marihuana está en todas las facultades y campus de la Universidad, siendo la Facultad de Educación la que presenta consumo de todas las sustancias.

\section{Religión, quintiles y consumo de drogas}

Nuestros resultados evidencian que a mayor ingreso económico familiar hay mayor consumo de drogas (Tabla 3). Los grupos de mayores ingresos consumen más drogas y experimentan más con una variedad de ellas.

Los estudiantes protestantes se distribuyen en los quintiles de menores ingresos y además tienen estilos de vida protectores hacia el consumo drogas (Tabla 4).

La religión del estudiante constituye un factor protector del consumo de drogas, sobre todo en el caso de las drogas ilícitas como marihuana y cocaína (Tabla 5). Como señalado anteriormente, resalta el hecho de que los estudiantes protestantes tiendan a consumir menos drogas que los estudiantes que participan en otras religiones. Los estudiantes católicos, respecto a las drogas lícitas, no se diferencian significativamente del consumo de los estudiantes que no se adscriben a creencias religiosas.

\section{Riesgos asociados al consumo de drogas}

El alcohol, la droga lícita más consumida por los estudiantes, es la droga que mayores problemas familiares y riesgos sociales les trae a los estudian- 
Tabla 2. Prevalencia de último año según Facultades

\begin{tabular}{|c|c|c|c|c|c|c|c|c|c|}
\hline \multirow[t]{2}{*}{ Facultad } & \multicolumn{9}{|c|}{ Prevalencia de último año (\%) } \\
\hline & Alcohol & $\begin{array}{l}\text { Mari- } \\
\text { huana }\end{array}$ & Tabaco & Cocaína & $\begin{array}{l}\text { Pasta } \\
\text { base }\end{array}$ & $\begin{array}{l}\text { Tranqui- } \\
\text { lizantes }\end{array}$ & $\begin{array}{l}\text { Inhalan- } \\
\text { tes }\end{array}$ & $\begin{array}{l}\text { Alucinó- } \\
\text { genos }\end{array}$ & $\begin{array}{l}\text { Estimu- } \\
\text { lantes }\end{array}$ \\
\hline $\begin{array}{l}\text { Arquitectura, } \\
\text { Urban. y Geograf. }\end{array}$ & 88,9 & 22,2 & 29,6 & & & & & & \\
\hline Cs. Biológicas & 95,2 & 28,6 & 33,3 & & & & & & \\
\hline $\begin{array}{l}\text { Cs. Económicas y } \\
\text { Administrativas }\end{array}$ & 84,1 & 11,6 & 30,4 & & & 7,2 & & & 1,4 \\
\hline $\begin{array}{l}\text { Ciencias Físicas y } \\
\text { Matemáticas }\end{array}$ & 78,6 & 10,7 & 14,3 & & 3,6 & & & & \\
\hline Cs. Forestales & 87,9 & 21,2 & 48,5 & 3,0 & & 9,1 & & & 3,0 \\
\hline $\begin{array}{l}\text { Cs. Jurídicas y } \\
\text { Sociales }\end{array}$ & 84,1 & 31,7 & 50,8 & 3,2 & & 3,2 & & 1,6 & 3,2 \\
\hline $\begin{array}{l}\text { Cs. Naturales y } \\
\text { Oceanográficas }\end{array}$ & 85,1 & 21,6 & 33,8 & 1,4 & 1,4 & 4,1 & & & 1,4 \\
\hline Cs. Químicas & 80,0 & 14,6 & 36,6 & 2,4 & 2,4 & 2,4 & & & 2,4 \\
\hline Cs. Sociales & 88,6 & 41,2 & 52,2 & 7,0 & 0,9 & 11,4 & & 1,8 & 5,3 \\
\hline Educación & 83,1 & 25,6 & 42,3 & 0,3 & 0,6 & 4,5 & 0,6 & 0,3 & 1,3 \\
\hline Farmacia & 90,5 & 23,8 & 28,6 & & & & 4,8 & 4,8 & 4,8 \\
\hline $\begin{array}{l}\text { Humanidades y } \\
\text { Artes }\end{array}$ & 80,0 & 23,3 & 56,7 & & & 10,0 & & & \\
\hline Ingeniería & 89,4 & 22,3 & 37,6 & 0,6 & 0,6 & 1,7 & 0,6 & & \\
\hline Medicina & 82,2 & 8,8 & 32,0 & & & 6,6 & & & 1,7 \\
\hline $\begin{array}{l}\text { Medicina } \\
\text { Veterinaria }\end{array}$ & 87,5 & 12,5 & 37,5 & & & & & & \\
\hline Odontología & 78,3 & 19,3 & 32,5 & 1,2 & & 2,4 & & 1,2 & 3,6 \\
\hline Campus Chillán & 87,0 & 21,7 & 47,8 & 2,9 & & & & & \\
\hline $\begin{array}{l}\text { Campus } \\
\text { Los Ángeles }\end{array}$ & 78,5 & 25,4 & 40,0 & 6,3 & 1,6 & 4,8 & 0,8 & & 1,6 \\
\hline
\end{tabular}

Tabla 3. Prevalencia de último año según quintiles

\begin{tabular}{|lrrrrr|}
\hline Substancia & \multicolumn{5}{c|}{$\begin{array}{c}\text { Prevalencia último año } \\
\text { (\% de los encuestados) }\end{array}$} \\
& Q1 & Q2 & Q3 & Q4 & Q5 \\
Marihuana & 19,0 & 19,7 & 21,3 & 27,8 & 29,6 \\
\hline Pasta Base & 0,6 & 0,5 & 0,4 & 0,6 & 0 \\
\hline Cocaína & 1,6 & 1,3 & 2,7 & 1,6 & 0,8 \\
$\begin{array}{l}\text { Tranquilizantes } \\
\text { s/receta }\end{array}$ & 5,9 & 3,4 & 4,1 & 5,5 & 5,0 \\
$\begin{array}{l}\text { Estimulantes } \\
\text { s/receta }\end{array}$ & 1,3 & 1,3 & 1,8 & 0,9 & 4,2 \\
Tabaco & 37,5 & 36,8 & 35,0 & 44,9 & 46,6 \\
\hline Alcohol & 80,2 & 81,3 & 86,9 & 88,1 & 93,1 \\
\hline
\end{tabular}

Tabla 4. Religión del encuestado por quintiles en porcentajes

\begin{tabular}{|lccccc|}
\hline Religión & \multicolumn{5}{c|}{$\begin{array}{l}\text { Ingreso familiar por quintil } \\
\text { (\% de los encuestados) }\end{array}$} \\
& Q1 & Q2 & Q3 & Q4 & Q5 \\
\hline Católico & 22,9 & 26,4 & 19,0 & 22,0 & 9,7 \\
\hline Protestante & 29,3 & 43,1 & 13,2 & 11,5 & 2,9 \\
\hline Otra religión & 20,8 & 37,5 & 14,6 & 20,8 & 6,3 \\
\hline Agnóstico & 31,3 & 16,9 & 12,0 & 28,9 & 10,8 \\
\hline Libre pensador & 18,2 & 25,0 & 16,2 & 29,9 & 10,4 \\
\hline $\begin{array}{l}\text { No pertenece a } \\
\text { ninguna religión }\end{array}$ & 19,0 & 30,2 & 11,9 & 28,6 & 10,3 \\
\hline Total & 23,3 & 28,8 & 16,5 & 22,6 & 89,0 \\
\hline
\end{tabular}


Consumo de drogas en universitarios - M. J. Sepúlveda et al

Tabla 5. Consumo de drogas por religión del encuestado

\begin{tabular}{|lcccccc|}
\hline Substancia & Católicos & Protestantes & $\begin{array}{c}\text { Consumo según Religión } \\
\text { Otras de los encuestados) }\end{array}$ & $\begin{array}{c}\text { (\% dibnósticos } \\
\text { religiones }\end{array}$ & $\begin{array}{c}\text { Libre } \\
\text { pensador }\end{array}$ & Sin religión \\
\hline Alcohol & 87,0 & 65,0 & 67,0 & 89,0 & 89,0 & 87,0 \\
\hline Tabaco & 41,0 & 23,0 & 31,0 & 47,0 & 48,0 & 41,0 \\
\hline Marihuana & 16,0 & 9,0 & 15,0 & 41,0 & 41,0 & 32,0 \\
Cocaína & 0,8 & 0,5 & 1,7 & 4,5 & 4,7 & 3,2 \\
\hline Tranquiliz. $s / r$ & 3,3 & 5,3 & 8,6 & 5,6 & 5,0 & 5,8 \\
\hline
\end{tabular}

$\mathrm{s} / \mathrm{r}=$ sin receta médica

Tabla 6. Riesgos por consumo de substancias en el último año

\begin{tabular}{|c|c|c|c|}
\hline \multirow[t]{2}{*}{$\begin{array}{l}\text { Situación problemática por el consumo de substancia en } \\
\text { los últimos } 12 \text { meses }\end{array}$} & \multicolumn{3}{|c|}{$\begin{array}{c}\text { Consumo } \\
\text { (\% de los encuestados) }\end{array}$} \\
\hline & Alcohol & Marihuana & Cocaína \\
\hline $\begin{array}{l}\text { Se ha expuesto a peligros contra su integridad física (a punto de chocar } \\
\text { en auto o sufrir cualquier otra clase de accidente) }\end{array}$ & 12,8 & 1,3 & 0,3 \\
\hline $\begin{array}{l}\text { Ha tenido problemas serios en casa, trabajo o universidad a causa del } \\
\text { consumo de esta substancia }\end{array}$ & 7,5 & 1,3 & 0,7 \\
\hline $\begin{array}{l}\text { Ha tenido problemas con la autoridad pública o con la ley a causa del } \\
\text { consumo de esta substancia (conducir vehículos en estado de ebriedad, } \\
\text { destruir equipamiento público, molestar a los vecinos, etc) }\end{array}$ & 19,7 & 2,3 & 0,6 \\
\hline $\begin{array}{l}\text { Ha tenido discusiones y disgustos serios con familiares y amigos que } \\
\text { resintieron su relación a causa del consumo de esta substancia }\end{array}$ & 16,6 & 1,8 & 0 \\
\hline $\begin{array}{l}\text { Se ha visto envuelto en alguna pelea o a agredido a alguien por el con- } \\
\text { sumo de esta substancia }\end{array}$ & 14,2 & 0 & 0,4 \\
\hline
\end{tabular}

tes. La marihuana es la droga ilícita más consumida por los estudiantes, sin embargo, se asocia muy poco a las conductas riesgosas (Tabla 6).

\section{Discusión}

Nuestro estudio reporta sobre el consumo de drogas en estudiantes universitarios con trayectoria académica, por lo que los resultados obtenidos están influenciados principalmente por la vida universitaria, las presiones académicas y las expectativas laborales. Al respecto, los estudios realizados en alumnos de primer año están influenciados por el cambio de escolar a universitario, la independencia familiar y la presión de los pares y los estudios realizados en población general entre 19 y 25 años incluye a universitarios, trabajadores y cesantes.
Los resultados presentados en este estudio demuestran que los estudiantes tienden a probar en el transcurso de su adolescencia y juventud una variedad de drogas de forma experimental, pero tienden a dejar de consumirla en época de estudios universitarios. Sin embargo, se evidenció que el alcohol es la droga lícita más consumida, sin distinción de sexo y la que mayores problemas familiares y riesgos sociales les ocasiona a los estudiantes. La droga ilícita más consumida por los estudiantes es la marihuana, sin embargo, se asocia muy poco a las conductas riesgosas.

El análisis revela que el consumo de alcohol y marihuana en estos estudiantes es mayor que los de la población chilena, tanto general como de la subpoblación de 19-25 años ${ }^{1}$ y otras poblaciones universitarias 3,5 y menor para tabaco, cocaína y pasta base. En el análisis por Facultad, 
nuestros resultados demostraron que en todas ellas, el consumo de alcohol y de marihuana son los que presentan la mayor prevalencia. El abuso de alcohol se ve favorecido por el estrés propio de una carga académica, al aumento de la oferta de venta de alcohol en las cercanías del campus universitario y el consumo de marihuana está es alto debido a la baja percepción de daño que existe con respecto a su uso.

Con respecto al tabaco, la tendencia a la baja de consumo en esta universidad se debería a la implementación precoz de una política de prohibición de fumar dentro de las dependencias universitarias. La tendencia a la baja de consumo de tabaco se observa también en otras universidades ${ }^{3,4}$ y se debería a las medidas de prevención instauradas a nivel mundial, apoyando fuertemente la tesis que la educación y la implementación de políticas de prevención masivas son las principales herramientas para la disminución del consumo de drogas.

Las cifras de consumo de cocaína y pasta base serían menores en los universitarios debido a una mayor percepción de daño con respecto a su uso.

Otro aporte de esta investigación fue la identificación de diferencias de género con respecto al consumo. Los hombres experimentan con más drogas y a más temprana edad que las mujeres. Cocaína, pasta base, éxtasis y alucinógenos, son consumidos preferentemente por hombres, mientras que las substancias de uso médico como tranquilizantes y analgésicos son consumidos preferentemente por mujeres.

Además, con este estudio se pudo pesquisar los factores que influenciarían el consumo de drogas. El ingreso económico familiar facilitaría el consumo de drogas en los estudiantes y la religión constituiría un factor protector del consumo de drogas. En este punto existirían dos aspectos relevantes a considerar a la hora de consumir o no drogas, a) uno relativo al costo económico y "moral" de perder una carrera a causa del consumo abusivo de las drogas, ya que la enseñanza superior se asocia a una movilidad social ascendente entre los estratos sociales bajos y medios, y b) la adscripción o creencia religiosa, la cual se establece como un factor protector del consumo de drogas, especialmente en quienes adhieren a la religión evangélica.

Por los resultados obtenidos y las características de la población, nos parece relevante enfatizar en las consecuencias académicas del consumo de dro- gas. Al respecto, está bien establecido que la corteza prefrontal está relacionada con la experiencia y la expresión emocional, con la capacidad para anticipar y planificar el futuro, la organización temporal de la conducta, el sentido de la responsabilidad hacia sí mismo y hacia los demás, la posibilidad de adaptarse a un entorno social complejo a costa de reprimir las propias tendencias instintivas, lo que podría resumirse por juicio ético y conducta social. El uso de marihuana se asocia con hipofunción de la corteza prefrontal, generando déficits en las funciones ejecutivas, en la atención, memoria, efectos que pueden durar más allá del período de consumo y ser acumulativos ${ }^{11,12}$. Esta hipofuncionalidad crea un abismo entre el conocimiento y el sentimiento perdiéndose la capacidad inhibidora sobre nuestros instintos más primarios apareciendo la desinhibición, aumento de la impulsividad y un comportamiento social inapropiado ${ }^{13,14}$. Esto aumentaría la determinación conductual por el sistema límbico, es decir, conductas guiadas por una gratificación inmediata sin contemplar consecuencias futuras.

Estos resultados, por tratarse de una muestra no probabilística, no pueden ser generalizables a la población total, sin embargo, nos permiten concluir que se requiere a la brevedad del fortalecimiento de políticas públicas orientadas a la prevención del consumo de drogas.

Agradecimientos: El presente trabajo de investigación contó con el apoyo de Vice-Rectoría, Proyecto de Docencia No 04-01de la Dirección de Docencia y CONACE.

\section{Referencias}

1. Consejo nacional para el control de estupefaciente (CONACE) (1994-2008). Estudio nacional sobre drogas en población general de Chile.

2. Ruangkanchanasetr S, Plitponkarnpim A, Hetrakul P, Kongsakon R. Youth risk behavior survey: Bangkok, Thailand. J Adolescent Health 2005; 36: 227-35.

3. Pillon S, O'brien B, Chávez K. The relationship between drugs use and risk behaviours in Brazilian university students. Rev Latinoam Enfermagem 2005; 13: 1169-76.

4. Boland M, Fitzpatrick P, Scallan E, Daly L, Herity B, Horgan J, et al. Trends in medical student use of tobacco, alcohol and drugs in an Irish university, 1973-2002. Drug Alcohol Depend 2006; 85: 123-8.

5. Jiménez-Muro FA, Belmonte San Agustín A, Marqueta 
Baile A, Gargallo Valero P, Nerín De La Puerta I. Consumo de drogas en estudiantes universitarios de primer curso. Adicciones 2009; 21: 21-8.

6. Rodríguez J, Fernández A, Hernández E, Valdés M, Villalón M, Ramírez S, et al. Estudio de prevalencia del consumo de drogas en escolares de Enseñanza Básica, a través de la metodología de pares. Rev Med Chile 2009; 137: 774-84.

7. Rodríguez J, Hernández E, Fernández A. Descripción del consumo de drogas lícitas e ilícitas por género a través de la metodología de pares. Rev Med Chile 2007; 135: 449-59.

8. Ramírez Castillo J, Cunningham J, Brands B, Strike C, Wright M. Normas percibidas por los estudiantes universitarios acerca de sus pares y uso de drogas en una universidad en Chile. Rev Latinoam Enfermagem 2009; 17: 907-12.

9. Romero M, Santander J, Hitschfeld M, Labbé M, Zamora V. Consumo de tabaco y alcohol entre los estudiantes de medicina de la Pontificia Universidad Católica de Chile. Rev Med Chile 2009; 137: 361-8.

10. Vivanco M. Diseño de muestras en investigación social. En: Canales, M, Coordinador, Metodología de Investigación Social: Introducción a los oficios. Santiago, Chile: LOM; 2006. p 141-67.

11. Lundqvist T. Imaging cognitive deficits in drug abuse. Curr. Top. Behav Neurosci 2010; 3: 247-75.

12. Burston J, Wiley J, Craig A, Selley D, Sim-Selley L. Regional enhancement of cannabinoid CB1; receptor desensitization in female adolescent rats following repeated Delta-tetrahydrocannabinol exposure. Br J Pharmacol 2010; 161: 103-12.

13. Damasio A. Emotion in the perspective of an integrated nervous system. Brain Research Reviews 1998; 26: 83-6.

14. Sánchez-Navarro J, Román F. Amígdala, corteza prefrontal y especialización hemisférica en la experiencia y expresión emocional. Anales de Psicología 2004; 20 : 223-40. 